\title{
Bioinformatics analysis of microarray data to identify the candidate biomarkers of lung adenocarcinoma
}

\author{
Tingting Guo ${ }^{\text {Equal first author, } 1}$, Hongtao Ma ${ }^{\text {Equal first author, } 1}$, Yubai Zhou ${ }^{\text {Corresp. } 1}$ \\ ${ }^{1}$ Department of Biotechnology, College of Life Science \& Bioengineering, Beijing University of Technology, Beijing, China \\ Corresponding Author: Yubai Zhou \\ Email address: zhouyubai@bjut.edu.cn
}

Background: Lung adenocarcinoma (LUAD) is the major subtype of lung cancer and the most lethal malignant disease worldwide. However, the molecular mechanisms underlying LUAD are not fully understood.

Methods: Four datasets GSE118370, GSE85841, GSE43458 and GSE32863 were obtained from the Gene Expression Omnibus (GEO). Identification of differentially expressed genes (DEGs) and functional enrichment analysis were performed using limma and clusterProfiler packages, respectively. Proteinprotein interaction (PPI) network was constructed via STRING database and the module analysis was performed by Cytoscape. Then, overall survival analysis was performed using Kaplan-Meier curve, and prognostic candidate biomarkers were further analyzed using oncomine database.

Results: Totally, 349 DEGs were identified, including 275 downregulated and 74 upregulated genes which were significantly enriched in the biological process of extracellular structure organization, leukocyte migration and response to peptide. The mainly enriched pathways were complement and coagulation cascades, malaria and prion diseases. By extracting key modules from the PPI network, 11 hub genes were screened out. Survival analysis showed that except VSIG4, other hub genes may be involved in the development of LUAD, in which MYH10, METTL7A, FCER1G and TMOD1 have not been reported previously to correlated with LUAD. Briefly, novel hub genes identified in this study will help to deepen our understanding of the molecular mechanisms of LUAD carcinogenesis and progression, and to discover candidate targets for early detection and treatment of LUAD. 
1 Bioinformatics analysis of microarray data to identify

2 the candidate biomarkers of lung adenocarcinoma

3

4

5

6

7

Tingting Guo, Hongtao Ma, Yubai Zhou

Department of Biotechnology, College of Life Science \& Bioengineering, Beijing University of Technology, Chaoyang, Beijing, China

Corresponding Author:

Yubai Zhou

Pingleyuan 100\#, District of Chaoyang, Beijing 100124, China.

Email address: zhouyubai@bjut.edu.cn

\section{Abstract}

Background: Lung adenocarcinoma (LUAD) is the major subtype of lung cancer and the most lethal malignant disease worldwide. However, the molecular mechanisms underlying LUAD are not fully understood.

Methods: Four datasets GSE118370, GSE85841, GSE43458 and GSE32863 were obtained from the Gene Expression Omnibus (GEO). Identification of differentially expressed genes (DEGs) and functional enrichment analysis were performed using limma and clusterProfiler packages, respectively. Protein-protein interaction (PPI) network was constructed via STRING database and the module analysis was performed by Cytoscape. Then, overall survival analysis was performed using Kaplan-Meier curve, and prognostic candidate biomarkers were further analyzed using oncomine database.

Results: Totally, 349 DEGs were identified, including 275 downregulated and 74 upregulated genes which were significantly enriched in the biological process of extracellular structure organization, leukocyte migration and response to peptide. The mainly enriched pathways were complement and coagulation cascades, malaria and prion diseases. By extracting key modules 
30 from the PPI network, 11 hub genes were screened out. Survival analysis showed that except 31 VSIG4, other hub genes may be involved in the development of LUAD, in which MYH10, 32 METTL7A, FCER1G and TMOD1 have not been reported previously to correlated with LUAD. 33 Briefly, novel hub genes identified in this study will help to deepen our understanding of the molecular mechanisms of LUAD carcinogenesis and progression, and to discover candidate targets for early detection and treatment of LUAD.

\section{Introduction}

Lung cancer remains the one of leading healthy issues worldwide, with estimated 2.1 million new cases and 1.8 million deaths in 2018(Bray et al. 2018). It has been ranked the first and second cancer morbidity of male and female in China respectively, and has the highest mortality rate (Sun et al. 2018). Lung adenocarcinoma (LUAD) is the most common subtype of lung cancer (Maemura et al. 2018; Walters et al. 2013). More than $60 \%$ of LUAD patients were observed harboring targetable gene alterations, which leading to remarkable responses in treating with tyrosine kinase inhibitors (TKIs), and associated with improved survival rate (Kris et al. 2014). Despite the substantial advance in combined therapies, the prognosis of LUAD is still dismal, the 5-year survival rate is not over 20\% (Chen et al. 2014b; Ettinger et al. 2013). Lacking sensitive and specific early biomarkers, high possibility of drug resistance and metastasis, is considered to contribute the high mortality of this disease. Therefore, there has a pressing need for identifying the more sensitive and specific biomarkers or drug targets of LUAD for developing effective diagnosis and treatment strategies.

Microarray technology provides an all-in-one system biology solution from hardware to software systems. It can simultaneously scan the hybridization signals of tens of thousands of gene probes in the chip and carry out quantitative analysis on the transcriptome profile of samples. Recent advances especially in the algorithms of probe signal detection and analysis, such as the introduction of artificial intelligence technologies, will make the results of microarray more accurate and reliable(Gan et al. 2019a; Gan et al. 2019b; Peng 2006). The microarray technique also provides a powerful tool for exploring the gene regulation pattern and molecular mechanisms 
57 involved in oncogenesis and progression of LUAD. Recently, different types of biomarkers

58 including coding genes, miRNAs, long non-coding RNAs and circRNAs have been identified in

59 lung cancer. Dysregulation of these molecules is involved in the tumor progression or is associated

60 with the prognosis of patients (Di et al. 2019; Vargas \& Harris 2016; Vencken et al. 2015; Wei \&

61 Zhou 2016). In view of the complexity of the molecular regulatory network of LUAD, current

62 studies on tumor biomarkers are not sufficient. Therefore, it is still necessary to identify novel

63 prognostic biomarkers, which will help us develop more sensitive and effective diagnostic and

64 therapeutic strategies. However, limited sample size and significant variability among different

65 projects make it hard to obtain credible results. In this study, four microarray datasets containing

66 mRNA expression data between LUAD and non-cancerous tissues were downloaded from GEO

67 and the DEGs were screened out. GO (Gene Ontology), KEGG (Kyoto Encyclopedia of Genes

68 and Genomes) and PPI network analyses were performed to explore the key modules and hub 69 genes involved in LUAD progression. In sum, 349 DEGs and 10 hub genes were screened out, 70 which may be candidate biomarkers for LUAD.

71

\section{Materials \& Methods}

\section{Data download and pre-processing}

74 Four datasets GSE118370, GSE32863, GSE85841 and GSE43458, which contain the gene 75 expression data of LUAD and normal tissues, were downloaded from GEO 76 (https://www.ncbi.nlm.nih.gov/geo/) by getGEO function in package GEOquery (Davis \& Meltzer 77 2007). The detail information of GEO datasets was listed in table 1 . The raw expression files of 78 four microarray datasets were pre-processed according to the method described previously with 79 minor modifications (Giulietti et al. 2016). Briefly, the CEL format files were input and 80 background correction and normalization were conducted using the Robust Multichip Average 81 (RMA) function implemented in affy package in R environment (Bolstad et al. 2003; Irizarry et 82 al. 2003). Next, the array probes were converted into matched gene symbols according to 83 annotation information. In case of multiple probes corresponding to a single gene, the value of 
84 gene expression was designated as the mean of the probes. Then, the batch effects among different

85

86

87

88

89

90

91

92

93

94

95

96

97

98

99

100

101

102

103

104

105

106

107

108

109

110

platforms were removed by ComBat function of sva package. Finally, the normalized microarraybased data of four datasets were merged into a single global dataset which contained a total of 12,926 common genes in all four GEO datasets.

\section{Identification of DEGs}

Identifying differentially expressed genes in different disease states and investigating their functions and interactions may help to unravel potential regulatory mechanisms for disease occurrence and progression. In present study, the DEGs between LUAD and normal tissues were identified by limma package (Ritchie et al. 2015). The Benjamini-Hochberg procedure was introduced to reduce the false positive rate (FDR) in multiple comparisons (Benjamini \& Hochberg 1995). Genes with $\mid \log 2$ Fold Change $\mid \geqslant 1$ and FDR $<0.05$ were considered as DEGs.

\section{GO and KEGG enrichment analysis}

GO and KEGG analyses were conducted using enrichGO and enrichKEGG functions of clusterProfiler package, respectively (Yu et al. 2012). p.adjust (FDR) $<0.05$ was considered to be statistically significant.

\section{Construction of PPI network and module analysis}

The network of proteins interaction provides valuable clues for understanding the molecular mechanisms underlying the progress of carcinoma. The PPI network was constructed by Search Tool for the Retrieval of Interacting Genes (STRING) (https://string-db.org/) with interaction score of 0.9 as the threshold. Subsequently, the candidate modules were detected by Cytoscape plugin molecular complex detection (MCODE) with default parameters: degree cut-off $=2$, node score cut-off $=0.2, \mathrm{k}$-core $=2$, and $\max$ depth $=100$.

\section{Hub gene analysis}

The seed genes in modules referred to hub genes. The overall survival analyses were performed using online tool Kaplan-Meier Plotter (http://kmplot.com/)(Gyorffy et al. 2013). The logrank $\mathrm{P}<0.05$ was considered statistically significant. The association of expression level of hub genes with clinical traits were analysis using Oncomine database(Rhodes et al. 2007).

Peer] reviewing PDF | (2019:04:36425:1:1:NEW 31 May 2019) 


\section{Results}

\section{Data preprocessing and DEG screening}

114 Four GEO datasets were downloaded, pre-processed and merged into a global dataset which 115 contained 112 LUAD and 102 normal samples. Totally, 349 DEGs were identified by limma 116 package (Ritchie et al. 2015), including 74 up-regulated genes and 275 down-regulated genes. The 117 most statistically significant up-regulated and down-regulated genes are listed in Table 2. The 118 distribution of DEGs was presented by volcano plot (Fig. 1).

\section{GO and KEGG analysis}

120 The biological functions and pathways analyses were conducted using $\mathrm{R}$ package clusterProfiler 121 (Yu et al. 2012). The GO categories of biological process (BP), cellular component (CC) and 122 molecular function (MF) were enriched respectively (Fig.2A-C) and the top 15 GO terms of the 123 up-regulated and down-regulated DEGs were listed in supplemental table 1,2 respectively. The 124 up-regulated DEGs were mainly associated with extracellular matrix processing, such as 125 extracellular matrix organization (BP, GO:0030198), extracellular matrix (CC, GO:0031012) and 126 serine-type endopeptidase activity (MF, GO:0004252). The down-regulated DEGs were most 127 significantly related to response to corticosteroid (BP, GO:0031960), extracellular matrix (CC, 128 GO:0031012) and growth factor binding (MF, GO:0019838). The DEGs were mainly enriched in 129 pathways of complement and coagulation cascades(hsa04610), malaria (hsa05144), prion 130 diseases(hsa05020), fluid shear stress and atherosclerosis(hsa05418), AGE-RAGE signaling 131 pathway in diabetic complications(hsa04933), vascular smooth muscle contraction (hsa04270), 132 IL-17 signaling pathway (hsa04657), leukocyte transendothelial migration (hsa04670), protein 133 digestion and absorption (hsa04974) and drug metabolism - cytochrome P450(hsa00982) (Table 3 134 and Fig. 2D).

\section{Construction of PPI network and module analysis}

136 PPI network reflect the spatiotemporal relationship of macromolecules within the cell which will 137 provide valuable information about molecular mechanisms in physiological and pathological 
138 process. To explore the molecular mechanisms underlying LUAD progression, online database 139 STRING was applied to construct the PPI network. The interaction score of 0.9 (highest 140 confidence) was set as threshold, and nodes without connections were removed from network.

141 Finally, the PPI network consisted of 349 nodes with 277 edges, and average local clustering 142 coefficient was 0.337(PPI enrichment p-value< 1.0E-16) (Fig. 3A). Then, the key modules were 143 identified via MCODE plugin. Eleven functional clusters of modules and related hub genes were 144 detected. The top 3 significant modules were presented in Fig. 3C-E. The KEGG analysis of 145 module genes revealed that the top 3 modules were mainly associated with chemokine signaling 146 pathway(hsa04062), complement and coagulation cascades(hsa04610), human cytomegalovirus 147 infection(hsa05163) and vascular smooth muscle contraction(hsa04270) (Fig. 3B).

\section{Hub genes analysis}

149 A total of 11 genes were identified as hub genes. The overall survival analysis of the hub genes 150 was performed using Kaplan-Meier curve. Except VSIG4, LUAD patients with downregulated 151 ADAMTS8, AOX1, EFEMP1, METTL7A, MYH10, PTGER4, TMOD1, CDH13 and upregulated 152 PRC1 showed worse overall survival (Fig. 4A-I). It is worth noting that FCER1G is downregulated 153 in LUAD patients, but the low expression level is associated with better overall survival $(\mathrm{HR}=$ 154 1.87) (Fig. 4J). Subsequently, the expression status of hub genes with $\mathrm{HR}<0.5$ or $\mathrm{HR}>2$ were 155 further validated using oncomine database. The result showed that ADAMTS8, METTL7A and 156 MYH10 were significantly downregulated and PRC1 was markedly overexpressed in LUAD in 157 the different datasets (Fig. 5). In the Okayama Lung dataset, the alternation of ADAMTS8, 158 METTL7A, MYH10 and PRC1 were associated with tumor grade (Fig. 6), implicating vital roles 159 of these genes in the carcinogenesis or progression of LUAD.

160

161 Discussion

162 In this study, four GEO datasets were analyzed and 349 DEGs were identified, including 74 up163 regulated and 275 down-regulated genes. The KEGG analysis revealed the top three enriched 164 pathways were complement and coagulation cascades, malaria and prion diseases. These 
165 annotation results provided valuable clues to reveal molecular interactions in the development of 166 LUAD. Indeed, the complement system has been reported to play critical roles in tumor 167 progression (Zhao et al. 2019). The overexpressed C3 in blood and downregulated C3 in tumor 168 tissues were observed in lung cancer patients, and related to poorer prognosis (Ajona et al. 2013; 169 Lin et al. 2014; Mehan et al. 2012). The confusing experimental results suggest that the 170 complement system may be involved in complex tumor regulatory processes by reshaping tumor 171 microenvironment, which is worthy of further study. In recent years, malaria and prion disease, 172 which had not previously attracted much attention, have also been found to be associated with 173 tumors. Epidemiological study has shown that the incidence of malaria is negatively correlated 174 with the mortality of colorectal cancer, breast cancer and lung cancer (Qin et al. 2017). The 175 proposed anti-tumor mechanisms included systemly stimulating the immune responses (Chen et 176 al. 2011) and inhibiting key pathways in tumor progress (Deng et al. 2018). Previously, prion 177 protein $(\mathrm{PrPc})$ was thought to play a role only in the central nervous system. However, 178 accumulating evidence shows that PrPc has wider biological functions that were not previously 179 expected (Mehrpour \& Codogno 2010). PrPc may be associated with the biology of many cancers, 180 and overexpression of PrPc promotes the proliferation, invasion and metastasis of the gastric 181 cancer cell(Liang et al. 2009; Mehrpour \& Codogno 2010; Pan et al. 2006). These data may 182 provide new ideas and directions for the mechanism research and therapeutic strategy of lung adenocarcinoma. The GO analyses indicated that DEGs were significantly related to biological

184 process of extracellular matrix organization and process. Previous studies have reported that 185 extracellular matrix $(\mathrm{ECM})$ remodeling promotes cancer progression and is associated with a poor 186 prognosis in lung cancer patients (Kopparam et al. 2017; Xia et al. 2012). The up- and downregulated DEGs were simultaneously enriched into extracellular matrix process, which is consistent with the propensity for metastasis and highly invasive characteristics of LUAD.

In line with the GO analysis, among 11 hub genes identified by PPI network and modules 190 analysis, EFEMP1, PTGER4, ADAMTS8, CDH13, MYH10 and METTL7A are associated with extracellular matrix process, and survival analysis indicated that down-regulation of these hub 
192 genes was associated with worse overall survival. EFEMP1 plays distinct biological functions in 193 different tumors. In osteosarcoma and gliomas, EFEMP1 is overexpressed and promotes the 194 invasion and metastasis of tumor cells in vitro and in vivo by activating the expression of MMP-2 195 and notch signaling, respectively(Hu et al. 2012; Hu et al. 2009; Wang et al. 2015), while in gastric 196 cancer, endometrial carcinoma, hepatocellular carcinoma and lung cancer, EFEMP1 is 197 downregulated, and is proposed as a prognosis biomarker(Chen et al. 2014a; Kim et al. 2014; 198 Nomoto et al. 2010; Yang et al. 2013; Yue et al. 2007; Zhu et al. 2014). Overexpression of 199 EFEMP1 has been reported to suppressed invasion and migration of LUAD cells via inhibiting the 200 epithelial-to-mesenchymal transition (EMT) pathway(Kim et al. 2014).PTGER4 is overexpressed 201 and proposed as a therapeutic target for LUAD and other cancers(Doherty et al. 2009; Fulton et 202 al. 2006; Heinrichs et al. 2018; Kim et al. 2010; Ma et al. 2006; Xin et al. 2012). The above report 203 is inconsistent with our results, which may be due to the heterogeneity of the tumor and the limited 204 number of samples. Therefore, subsequent large sample functional verification is required. 205 ADAMTS8 encodes an inactive proenzyme and forms mature active enzyme by proteolysis(Apte 206 2009). ADAMTS8 is identified as a secretory angiogenesis inhibitor that inhibits VEGF-mediated 207 angiogenesis by blocking the EGFR signaling pathway(Choi et al. 2014; Dunn et al. 2006; 208 Vazquez et al. 1999). Downregulated ADAMTS8 in some cancers including NSCLC (Heighway 209 et al. 2002; Huang et al. 2019; Masui et al. 2001; Porter et al. 2004; Rodriguez-Rodero et al. 2013; 210 Zhao et al. 2018) is associated with poorer prognosis (Drilon et al. 2014; Li et al. 2015; Porter et 211 al. 2006). CDH13 encodes a member of cadherin superfamily. The hypermethylation in promoter 212 region of $\mathrm{CDH} 13$ was frequently observed in lung cancer, and proposed to correlate to drug 213 sensitivity and poorer prognosis (Kontic et al. 2012; Toyooka et al. 2006; Zhai \& Li 2014; Zhong 214 et al. 2015). As the only upregulated hub gene, PRC1 is involved in the process of cytokinesis and 215 is upregulated in breast cancer, hepatocellular carcinoma, and lung cancer(Jiang et al. 1998; Liu 216 et al. 2018; Shimo et al. 2007; Zhan et al. 2017a). Recent research has found that it promoted 217 proliferation and metastasis of lung adenocarcinoma cells via potentiating the Wnt/ $\beta$-catenin 
218 pathway(Zhan et al. 2017b), and inhibiting the expression of PRC1 in LUAD cells by miR-1-3p 219 was reported to suppress tumorigenesis ( $\mathrm{Li}$ et al. 2019).

220 Literature retrieval showed that the relation of LUAD and hub genes MYH10, METTL7A, 221 FCER1G and TMOD1 has not been reported. MYH10 belongs to the myosin superfamily, which 222 can regulate ECM remodeling (Kim et al. 2018; Kim et al. 2015). Methyltransferase METTL7A 223 was found to facilitate Hepatitis C Virus (HCV) propagation via recruitment of NS4B (Park et al. 224 2015). FCER1G encodes a high affinity IgE receptor which is associated with prognosis of renal 225 clear cell carcinoma (Chen et al. 2017). As an aldehyde oxidase, TMOD1 is an actin-capping 226 protein involved in regulation of the length and depolymerization of actin filaments. 227 Overexpression of TMOD1 promotes cell proliferation of breast cancers and metastasis of oral 228 squamous cell carcinoma (Ito-Kureha et al. 2015; Suzuki et al. 2016). However, the molecular 229 mechanism of these newly identified hub genes in LUAD remains largely unknown, and further 230 functional studies were warranted.

231 In present study, we have identified a set of candidate biomarkers that may play an important 232 role in the progression of LUAD. These newly identified hub genes could be used as research 233 subjects for exploring their roles in the disease process, so as to further deepen our understanding 234 of the molecular mechanism of LUAD, and also as potential prognostic biomarkers for clinical 235 validation studies to clarify their prognostic effects. However, there are still some limitations in 236 this study. First, this study is based on bioinformatics analysis of published data and lacks 237 experimental verification, and the study cannot determine whether there is a causal relationship 238 between the differential expression of hub genes and disease progression. Finally, although we 239 combined four GEO datasets, the number of samples is still relatively small, which may lead to 240 potential unreliable results. Therefore, subsequent bioinformatics analysis and experimental 241 verification with larger samples are necessary.

\section{Conclusions}


244 In summary, this study identified several differentially expressed genes by integrating four GEO

245 datasets and extracted 11 hub genes from PPI network, among which 10 hub genes were shown to

246 be related to the occurrence and development of lung adenocarcinoma and 4 hub genes have not

247 been previously reported but may play an important role in LUAD. The molecular mechanism of

248 these novel hub genes in LUAD is worthy of further study, and relevant prognostic model can also

249 be constructed based on these genes for risk assessment, classification and prognostic judgment of

250 patients with LUAD.

251

252

\section{References}

253

254

255

256

257

258

259

260

261

262

263

264

265

266

267

268

269

270

271

272

273

274

275

276

277

278

279

280

Ajona D, Pajares MJ, Corrales L, Perez-Gracia JL, Agorreta J, Lozano MD, Torre W, Massion PP, de-Torres JP, Jantus-Lewintre E, Camps C, Zulueta JJ, Montuenga LM, and Pio R. 2013. Investigation of complement activation product c4d as a diagnostic and prognostic biomarker for lung cancer. J Natl Cancer Inst 105:13851393. 10.1093/jnci/djt205

Apte SS. 2009. A disintegrin-like and metalloprotease (reprolysin-type) with thrombospondin type 1 motif (ADAMTS) superfamily: functions and mechanisms. $J$ Biol Chem 284:31493-31497. 10.1074/jbc.R109.052340

Benjamini Y, and Hochberg Y. 1995. Controlling the false discovery rate: a practical and powerful approach to multiple testing. Journal of the Royal statistical society: series B 57:289-300.

Bolstad BM, Irizarry RA, Astrand M, and Speed TP. 2003. A comparison of normalization methods for high density oligonucleotide array data based on variance and bias. Bioinformatics 19:185-193. 10.1093/bioinformatics/19.2.185

Bray F, Ferlay J, Soerjomataram I, Siegel RL, Torre LA, and Jemal A. 2018. Global cancer statistics 2018: GLOBOCAN estimates of incidence and mortality worldwide for 36 cancers in 185 countries. CA Cancer J Clin 68:394-424. 10.3322/caac.21492

Chen L, He Z, Qin L, Li Q, Shi X, Zhao S, Chen L, Zhong N, and Chen X. 2011. Antitumor effect of malaria parasite infection in a murine Lewis lung cancer model through induction of innate and adaptive immunity. PLoS One 6:e24407. 10.1371/journal.pone.0024407

Chen L, Yuan L, Wang Y, Wang G, Zhu Y, Cao R, Qian G, Xie C, Liu X, Xiao Y, and Wang X. 2017. Co-expression network analysis identified FCER1G in association with progression and prognosis in human clear cell renal cell carcinoma. Int J Biol Sci 13:1361-1372. 10.7150/ijbs.21657

Chen X, Meng J, Yue W, Yu J, Yang J, Yao Z, and Zhang L. 2014a. Fibulin-3 suppresses Wnt/beta-catenin signaling and lung cancer invasion. Carcinogenesis 35:1707-1716. 10.1093/carcin/bgu023

Chen Z, Fillmore CM, Hammerman PS, Kim CF, and Wong KK. 2014b. Non-small-cell lung cancers: a heterogeneous set of diseases. Nat Rev Cancer 14:535-546. 10.1038/nrc3775

Choi GC, Li J, Wang Y, Li L, Zhong L, Ma B, Su X, Ying J, Xiang T, Rha SY, Yu J, Sung JJ, Tsao SW, Chan AT, and Tao Q. 2014. The metalloprotease ADAMTS8 displays antitumor properties through antagonizing EGFR-MEK-ERK signaling and is silenced in carcinomas by CpG methylation. Mol Cancer Res 12:228-

Peer] reviewing PDF | (2019:04:36425:1:1:NEW 31 May 2019) 
281

282

283

284

285

286

287

288

289

290

291

292

293

294

295

296

297

298

299

300

301

302

303

304

305

306

307

308

309

310

311

312

313

314

315

316

317

318

319

320

321

238. 10.1158/1541-7786.Mcr-13-0195

Davis S, and Meltzer PS. 2007. GEOquery: a bridge between the Gene Expression Omnibus (GEO) and BioConductor. Bioinformatics 23:1846-1847. 10.1093/bioinformatics/btm254

Deng XF, Zhou D, Liu QX, Zheng H, Ding Y, Xu WY, Min JX, and Dai JG. 2018. Plasmodium circumsporozoite protein suppresses the growth of A549 cells via inhibiting nuclear transcription factor kappaB. Oncol Lett 15:6585-6591. 10.3892/ol.2018.8115

Di X, Jin X, Li R, Zhao M, and Wang K. 2019. CircRNAs and lung cancer: Biomarkers and master regulators. Life Sci 220:177-185. 10.1016/j.lfs.2019.01.055

Doherty GA, Byrne SM, Molloy ES, Malhotra V, Austin SC, Kay EW, Murray FE, and Fitzgerald DJ. 2009. Proneoplastic effects of PGE2 mediated by EP4 receptor in colorectal cancer. BMC Cancer 9:207. 10.1186/1471-2407-9-207

Drilon A, Sugita H, Sima CS, Zauderer M, Rudin CM, Kris MG, Rusch VW, and Azzoli CG. 2014. A prospective study of tumor suppressor gene methylation as a prognostic biomarker in surgically resected stage I to IIIA non-small-cell lung cancers. $J$ Thorac Oncol 9:1272-1277. 10.1097/jto.0000000000000256

Dunn JR, Reed JE, du Plessis DG, Shaw EJ, Reeves P, Gee AL, Warnke P, and Walker C. 2006. Expression of ADAMTS-8, a secreted protease with antiangiogenic properties, is downregulated in brain tumours. $\mathrm{Br} J$ Cancer 94:1186-1193. 10.1038/sj.bjc.6603006

Ettinger DS, Akerley W, Borghaei H, Chang AC, Cheney RT, Chirieac LR, D'Amico TA, Demmy TL, Govindan R, Grannis FW, Jr., Grant SC, Horn L, Jahan TM, Komaki R, Kong FM, Kris MG, Krug LM, Lackner RP, Lennes IT, Loo BW, Jr., Martins R, Otterson GA, Patel JD, Pinder-Schenck MC, Pisters KM, Reckamp K, Riely GJ, Rohren E, Shapiro TA, Swanson SJ, Tauer K, Wood DE, Yang SC, Gregory K, and Hughes M. 2013. Non-small cell lung cancer, version 2.2013. J Natl Compr Canc Netw 11:645-653; quiz 653.

Fulton AM, Ma X, and Kundu N. 2006. Targeting prostaglandin E EP receptors to inhibit metastasis. Cancer Res 66:9794-9797. 10.1158/0008-5472.Can-06-2067

Gan Z, Zeng N, Zou F, Chen J, Du M, Liao L-C, Li H, and Zhang Y. 2019a. Multilevel Segmentation Optimized by Physical Information for Gridding of Microarray Images. Ieee Access 7:32146-32153.

Gan Z, Zou F, Zeng N, Xiong B, Liao L, Li H, Luo X, and Du M. 2019b. Wavelet Denoising Algorithm based on NDOA Compressed Sensing for Fluorescence Image of Microarray. Ieee Access 7:13338-13346. http://10.0.4.85/ACCESS.2019.2891759

Giulietti M, Occhipinti G, Principato G, and Piva F. 2016. Weighted gene co-expression network analysis reveals key genes involved in pancreatic ductal adenocarcinoma development. Cell Oncol (Dordr) 39:379-388. 10.1007/s13402-016-0283-7

Gyorffy B, Surowiak P, Budczies J, and Lanczky A. 2013. Online survival analysis software to assess the prognostic value of biomarkers using transcriptomic data in non-small-cell lung cancer. PLoS One 8:e82241. 10.1371/journal.pone.0082241

Heighway J, Knapp T, Boyce L, Brennand S, Field JK, Betticher DC, Ratschiller D, Gugger M, Donovan M, Lasek A, and Rickert P. 2002. Expression profiling of primary non-small cell lung cancer for target identification. Oncogene 21:7749-7763. 10.1038/sj.onc.1205979

Heinrichs SKM, Hess T, Becker J, Hamann L, Vashist YK, Butterbach K, Schmidt T, Alakus H, Krasniuk I, Hoblinger A, Lingohr P, Ludwig M, Hagel AF, Schildberg CW, Veits L, Gyvyte U, Weise K, Schuller V, Bohmer AC, Schroder J, Gehlen J, Kreuser N, Hofer S, Lang H, Lordick F, Malfertheiner P, Moehler M, Pech O, Vassos 
N, Rodermann E, Izbicki JR, Kruschewski M, Ott K, Schumann RR, Vieth M, Mangold E, Gasenko E, Kupcinskas L, Brenner H, Grimminger P, Bujanda L, Sopena F, Espinel J, Thomson C, Perez-Aisa A, Campo R, Geijo F, Collette D, Bruns C, Messerle K, Gockel I, Nothen MM, Lippert H, Ridwelski K, Lanas A, Keller G, Knapp M, Leja M, Kupcinskas J, Garcia-Gonzalez MA, Venerito M, and Schumacher J. 2018. Evidence for PTGER4, PSCA, and MBOAT7 as risk genes for gastric cancer on the genome and transcriptome level. Cancer Med 7:5057-5065. 10.1002/cam4.1719

Hu B, Nandhu MS, Sim H, Agudelo-Garcia PA, Saldivar JC, Dolan CE, Mora ME, Nuovo GJ, Cole SE, and Viapiano MS. 2012. Fibulin-3 promotes glioma growth and resistance through a novel paracrine regulation of Notch signaling. Cancer Res 72:3873-3885. 10.1158/0008-5472.Can-12-1060

$\mathrm{Hu}$ B, Thirtamara-Rajamani KK, Sim H, and Viapiano MS. 2009. Fibulin-3 is uniquely upregulated in malignant gliomas and promotes tumor cell motility and invasion. Mol Cancer Res 7:1756-1770. 10.1158/15417786.Mcr-09-0207

Huang J, Sun Y, Chen H, Liao Y, Li S, Chen C, and Yang Z. 2019. ADAMTS5 acts as a tumor suppressor by inhibiting migration, invasion and angiogenesis in human gastric cancer. Gastric Cancer 22:287-301. 10.1007/s10120018-0866-2

Irizarry RA, Hobbs B, Collin F, Beazer-Barclay YD, Antonellis KJ, Scherf U, and Speed TP. 2003. Exploration, normalization, and summaries of high density oligonucleotide array probe level data. Biostatistics 4:249-264. 10.1093/biostatistics/4.2.249

Ito-Kureha T, Koshikawa N, Yamamoto M, Semba K, Yamaguchi N, Yamamoto T, Seiki M, and Inoue J. 2015. Tropomodulin 1 expression driven by NF-kappaB enhances breast cancer growth. Cancer Res 75:62-72. 10.1158/0008-5472.Can-13-3455

Jiang W, Jimenez G, Wells NJ, Hope TJ, Wahl GM, Hunter T, and Fukunaga R. 1998. PRC1: a human mitotic spindleassociated CDK substrate protein required for cytokinesis. Mol Cell 2:877-885.

Kim HT, Yin W, Jin YJ, Panza P, Gunawan F, Grohmann B, Buettner C, Sokol AM, Preussner J, Guenther S, Kostin S, Ruppert C, Bhagwat AM, Ma X, Graumann J, Looso M, Guenther A, Adelstein RS, Offermanns S, and Stainier DYR. 2018. Myh10 deficiency leads to defective extracellular matrix remodeling and pulmonary disease. Nat Commun 9:4600. 10.1038/s41467-018-06833-7

Kim IG, Kim SY, Choi SI, Lee JH, Kim KC, and Cho EW. 2014. Fibulin-3-mediated inhibition of epithelial-tomesenchymal transition and self-renewal of ALDH+ lung cancer stem cells through IGF1R signaling. Oncogene 33:3908-3917. 10.1038/onc.2013.373

Kim JI, Lakshmikanthan V, Frilot N, and Daaka Y. 2010. Prostaglandin E2 promotes lung cancer cell migration via EP4-betaArrestin1-c-Src signalsome. Mol Cancer Res 8:569-577. 10.1158/1541-7786.Mcr-09-0511

Kim JS, Kurie JM, and Ahn YH. 2015. BMP4 depletion by miR-200 inhibits tumorigenesis and metastasis of lung adenocarcinoma cells. Mol Cancer 14:173. 10.1186/s12943-015-0441-y

Kontic M, Stojsic J, Jovanovic D, Bunjevacki V, Ognjanovic S, Kuriger J, Puumala S, and Nelson HH. 2012. Aberrant promoter methylation of CDH13 and MGMT genes is associated with clinicopathologic characteristics of primary non-small-cell lung carcinoma. Clin Lung Cancer 13:297-303. 10.1016/j.cllc.2011.11.003

Kopparam J, Chiffelle J, Angelino P, Piersigilli A, Zangger N, Delorenzi M, and Meylan E. 2017. RIP4 inhibits STAT3 signaling to sustain lung adenocarcinoma differentiation. Cell Death Differ 24:1761-1771. $10.1038 /$ cdd.2017.81

Kris MG, Johnson BE, Berry LD, Kwiatkowski DJ, Iafrate AJ, Wistuba, II, Varella-Garcia M, Franklin WA, Aronson 
363

364

365

366

367

368

369

370

371

372

373

374

375

376

377

378

379

380

381

382

383

384

385

386

387

388

389

390

391

392

393

394

395

396

397

398

399

400

401

402

403
SL, Su PF, Shyr Y, Camidge DR, Sequist LV, Glisson BS, Khuri FR, Garon EB, Pao W, Rudin C, Schiller J, Haura EB, Socinski M, Shirai K, Chen H, Giaccone G, Ladanyi M, Kugler K, Minna JD, and Bunn PA. 2014. Using multiplexed assays of oncogenic drivers in lung cancers to select targeted drugs. JAMA 311:1998-2006. 10.1001/jama.2014.3741

Leek JT, Johnson WE, Parker HS, Jaffe AE, and Storey JD. 2012. The sva package for removing batch effects and other unwanted variation in high-throughput experiments. Bioinformatics 28:882-883. 10.1093/bioinformatics/bts034

Li C, Xiong Y, Yang X, Wang L, Zhang S, Dai N, Li M, Ren T, Yang Y, Zhou SF, Gan L, and Wang D. 2015. Lost expression of ADAMTS5 protein associates with progression and poor prognosis of hepatocellular carcinoma. Drug Des Devel Ther 9:1773-1783. 10.2147/dddt.S77069

Li T, Wang X, Jing L, and Li Y. 2019. MiR-1-3p Inhibits Lung Adenocarcinoma Cell Tumorigenesis via Targeting Protein Regulator of Cytokinesis 1. Front Oncol 9:120. 10.3389/fonc.2019.00120

Liang J, Wang J, Luo G, Pan Y, Wang X, Guo C, Zhang D, Yin F, Zhang X, Liu J, Wang J, Guo X, Wu K, and Fan D. 2009. Function of PrPC (1-OPRD) in biological activities of gastric cancer cell lines. J Cell Mol Med 13:4453-4464. 10.1111/j.1582-4934.2009.00687.x

Lin K, He S, He L, Chen J, Cheng X, Zhang G, and Zhu B. 2014. Complement component 3 is a prognostic factor of nonsmall cell lung cancer. Mol Med Rep 10:811-817. 10.3892/mmr.2014.2230

Liu X, Li Y, Meng L, Liu XY, Peng A, Chen Y, Liu C, Chen H, Sun S, Miao X, Zhang Y, Zheng L, and Huang K. 2018. Reducing protein regulator of cytokinesis 1 as a prospective therapy for hepatocellular carcinoma. Cell Death Dis 9:534. 10.1038/s41419-018-0555-4

Ma X, Kundu N, Rifat S, Walser T, and Fulton AM. 2006. Prostaglandin E receptor EP4 antagonism inhibits breast cancer metastasis. Cancer Res 66:2923-2927. 10.1158/0008-5472.Can-05-4348

Maemura K, Watanabe K, Ando T, Hiyama N, Sakatani T, Amano Y, Kage H, Nakajima J, Yatomi Y, Nagase T, and Takai D. 2018. Altered editing level of microRNAs is a potential biomarker in lung adenocarcinoma. Cancer Sci 109:3326-3335. 10.1111/cas.13742

Masui T, Hosotani R, Tsuji S, Miyamoto Y, Yasuda S, Ida J, Nakajima S, Kawaguchi M, Kobayashi H, Koizumi M, Toyoda E, Tulachan S, Arii S, Doi R, and Imamura M. 2001. Expression of METH-1 and METH-2 in pancreatic cancer. Clin Cancer Res 7:3437-3443.

Mehan MR, Ayers D, Thirstrup D, Xiong W, Ostroff RM, Brody EN, Walker JJ, Gold L, Jarvis TC, Janjic N, Baird GS, and Wilcox SK. 2012. Protein signature of lung cancer tissues. PLoS One 7:e35157. 10.1371/journal.pone.0035157

Mehrpour M, and Codogno P. 2010. Prion protein: From physiology to cancer biology. Cancer Lett 290:1-23. 10.1016/j.canlet.2009.07.009

Nomoto S, Kanda M, Okamura Y, Nishikawa Y, Qiyong L, Fujii T, Sugimoto H, Takeda S, and Nakao A. 2010. Epidermal growth factor-containing fibulin-like extracellular matrix protein 1, EFEMP1, a novel tumorsuppressor gene detected in hepatocellular carcinoma using double combination array analysis. Ann Surg Oncol 17:923-932. 10.1245/s10434-009-0790-0

Pan Y, Zhao L, Liang J, Liu J, Shi Y, Liu N, Zhang G, Jin H, Gao J, Xie H, Wang J, Liu Z, and Fan D. 2006. Cellular prion protein promotes invasion and metastasis of gastric cancer. FASEB $J$ 20:1886-1888. 10.1096/fj.066138fje

Park EM, Lim YS, Ahn BY, and Hwang SB. 2015. AAM-B Interacts with Nonstructural 4B and Regulates Hepatitis 
404

405

406

407

408

409

410

411

412

413

414

415

416

417

418

419

420

421

422

423

424

425

426

427

428

429

430

431

432

433

434

435

436

437

438

439

440

441

442

443

444

C Virus Propagation. PLoS One 10:e0132839. 10.1371/journal.pone.0132839

Peng Y. 2006. A novel ensemble machine learning for robust microarray data classification. Comput Biol Med 36:553573. 10.1016/j.compbiomed.2005.04.001

Porter S, Scott SD, Sassoon EM, Williams MR, Jones JL, Girling AC, Ball RY, and Edwards DR. 2004. Dysregulated expression of adamalysin-thrombospondin genes in human breast carcinoma. Clin Cancer Res 10:2429-2440.

Porter S, Span PN, Sweep FC, Tjan-Heijnen VC, Pennington CJ, Pedersen TX, Johnsen M, Lund LR, Romer J, and Edwards DR. 2006. ADAMTS8 and ADAMTS15 expression predicts survival in human breast carcinoma. Int J Cancer 118:1241-1247. 10.1002/ijc.21476

Qin L, Chen C, Chen L, Xue R, Ou-Yang M, Zhou C, Zhao S, He Z, Xia Y, He J, Liu P, Zhong N, and Chen X. 2017. Worldwide malaria incidence and cancer mortality are inversely associated. Infect Agent Cancer 12:14. 10.1186/s13027-017-0117-x

Rhodes DR, Kalyana-Sundaram S, Mahavisno V, Varambally R, Yu J, Briggs BB, Barrette TR, Anstet MJ, KinceadBeal C, Kulkarni P, Varambally S, Ghosh D, and Chinnaiyan AM. 2007. Oncomine 3.0: genes, pathways, and networks in a collection of 18,000 cancer gene expression profiles. Neoplasia 9:166-180.

Ritchie ME, Phipson B, Wu D, Hu Y, Law CW, Shi W, and Smyth GK. 2015. limma powers differential expression analyses for RNA-sequencing and microarray studies. Nucleic Acids Res 43:e47. 10.1093/nar/gkv007

Rodriguez-Rodero S, Fernandez AF, Fernandez-Morera JL, Castro-Santos P, Bayon GF, Ferrero C, Urdinguio RG, Gonzalez-Marquez R, Suarez C, Fernandez-Vega I, Fresno Forcelledo MF, Martinez-Camblor P, Mancikova V, Castelblanco E, Perez M, Marron PI, Mendiola M, Hardisson D, Santisteban P, Riesco-Eizaguirre G, Matias-Guiu X, Carnero A, Robledo M, Delgado-Alvarez E, Menendez-Torre E, and Fraga MF. 2013. DNA methylation signatures identify biologically distinct thyroid cancer subtypes. J Clin Endocrinol Metab 98:2811-2821. 10.1210/jc.2012-3566

Shimo A, Nishidate T, Ohta T, Fukuda M, Nakamura Y, and Katagiri T. 2007. Elevated expression of protein regulator of cytokinesis 1, involved in the growth of breast cancer cells. Cancer Sci 98:174-181. 10.1111/j.13497006.2006.00381.x

Sun KX, Zheng RS, Zeng HM, Zhang SW, Zou XN, Gu XY, Xia CF, Yang ZX, Li H, Chen WQ, and He J. 2018. [The incidence and mortality of lung cancer in China, 2014]. Zhonghua Zhong Liu Za Zhi 40:805-811. 10.3760/cma.j.issn.0253-3766.2018.11.002

Suzuki T, Kasamatsu A, Miyamoto I, Saito T, Higo M, Endo-Sakamoto Y, Shiiba M, Tanzawa H, and Uzawa K. 2016. Overexpression of TMOD1 is associated with enhanced regional lymph node metastasis in human oral cancer. Int J Oncol 48:607-612. 10.3892/ijo.2015.3305

Toyooka S, Tokumo M, Shigematsu H, Matsuo K, Asano H, Tomii K, Ichihara S, Suzuki M, Aoe M, Date H, Gazdar AF, and Shimizu N. 2006. Mutational and epigenetic evidence for independent pathways for lung adenocarcinomas arising in smokers and never smokers. Cancer Res 66:1371-1375. 10.1158/0008-5472.Can05-2625

Vargas AJ, and Harris CC. 2016. Biomarker development in the precision medicine era: lung cancer as a case study. Nat Rev Cancer 16:525-537. 10.1038/nrc.2016.56

Vazquez F, Hastings G, Ortega MA, Lane TF, Oikemus S, Lombardo M, and Iruela-Arispe ML. 1999. METH-1, a human ortholog of ADAMTS-1, and METH-2 are members of a new family of proteins with angio-inhibitory activity. $J$ Biol Chem 274:23349-23357.

Vencken SF, Greene CM, and McKiernan PJ. 2015. Non-coding RNA as lung disease biomarkers. Thorax 70:501-

Peer] reviewing PDF | (2019:04:36425:1:1:NEW 31 May 2019) 
445

446

447

448

449

450

451

452

453

454

455

456

457

458

459

460

461

462

463

464

465

466

467

468

469

470

471

472

473

474

475

476

477

478

479

480

481

482

483

484

485

503. 10.1136/thoraxjnl-2014-206193

Walters S, Maringe C, Coleman MP, Peake MD, Butler J, Young N, Bergstrom S, Hanna L, Jakobsen E, Kolbeck K, Sundstrom S, Engholm G, Gavin A, Gjerstorff ML, Hatcher J, Johannesen TB, Linklater KM, McGahan CE, Steward J, Tracey E, Turner D, Richards MA, and Rachet B. 2013. Lung cancer survival and stage at diagnosis in Australia, Canada, Denmark, Norway, Sweden and the UK: a population-based study, 20042007. Thorax 68:551-564. 10.1136/thoraxjnl-2012-202297

Wang Z, Cao CJ, Huang LL, Ke ZF, Luo CJ, Lin ZW, Wang F, Zhang YQ, and Wang LT. 2015. EFEMP1 promotes the migration and invasion of osteosarcoma via MMP-2 with induction by AEG-1 via NF-kappaB signaling pathway. Oncotarget 6:14191-14208. 10.18632/oncotarget.3691

Wei MM, and Zhou GB. 2016. Long Non-coding RNAs and Their Roles in Non-small-cell Lung Cancer. Genomics Proteomics Bioinformatics 14:280-288. 10.1016/j.gpb.2016.03.007

Xia Y, Yeddula N, Leblanc M, Ke E, Zhang Y, Oldfield E, Shaw RJ, and Verma IM. 2012. Reduced cell proliferation by IKK2 depletion in a mouse lung-cancer model. Nat Cell Biol 14:257-265. 10.1038/ncb2428

Xin X, Majumder M, Girish GV, Mohindra V, Maruyama T, and Lala PK. 2012. Targeting COX-2 and EP4 to control tumor growth, angiogenesis, lymphangiogenesis and metastasis to the lungs and lymph nodes in a breast cancer model. Lab Invest 92:1115-1128. 10.1038/labinvest.2012.90

Yang T, Qiu H, Bao W, Li B, Lu C, Du G, Luo X, Wang L, and Wan X. 2013. Epigenetic inactivation of EFEMP1 is associated with tumor suppressive function in endometrial carcinoma. PLoS One 8:e67458. 10.1371/journal.pone.0067458

Yu G, Wang LG, Han Y, and He QY. 2012. clusterProfiler: an R package for comparing biological themes among gene clusters. OMICS 16:284-287. 10.1089/omi.2011.0118

Yue W, Dacic S, Sun Q, Landreneau R, Guo M, Zhou W, Siegfried JM, Yu J, and Zhang L. 2007. Frequent inactivation of RAMP2, EFEMP1 and Dutt1 in lung cancer by promoter hypermethylation. Clin Cancer Res 13:43364344. 10.1158/1078-0432.Ccr-07-0015

Zhai X, and Li SJ. 2014. Methylation of RASSF1A and CDH13 genes in individualized chemotherapy for patients with non-small cell lung cancer. Asian Pac J Cancer Prev 15:4925-4928.

Zhan P, Xi GM, Liu HB, Liu YF, Xu WJ, Zhu Q, Zhou ZJ, Miao YY, Wang XX, Jin JJ, Lv TF, and Song Y. 2017 a. Protein regulator of cytokinesis-1 expression: prognostic value in lung squamous cell carcinoma patients. $J$ Thorac Dis 9:2054-2060. 10.21037/jtd.2017.06.91

Zhan P, Zhang B, Xi GM, Wu Y, Liu HB, Liu YF, Xu WJ, Zhu QQ, Cai F, Zhou ZJ, Miu YY, Wang XX, Jin JJ, Li Q, Qian LP, Lv TF, and Song Y. 2017b. PRC1 contributes to tumorigenesis of lung adenocarcinoma in association with the Wnt/beta-catenin signaling pathway. Mol Cancer 16:108. 10.1186/s12943-017-0682-z

Zhao P, Wu J, Lu F, Peng X, Liu C, Zhou N, and Ying M. 2019. The imbalance in the complement system and its possible physiological mechanisms in patients with lung cancer. BMC Cancer 19:201. 10.1186/s12885-0195422-X

Zhao X, Yang C, Wu J, and Nan Y. 2018. ADAMTS8 targets ERK to suppress cell proliferation, invasion, and metastasis of hepatocellular carcinoma. Onco Targets Ther 11:7569-7578. 10.2147/ott.S173360

Zhong YH, Peng H, Cheng HZ, and Wang P. 2015. Quantitative assessment of the diagnostic role of CDH13 promoter methylation in lung cancer. Asian Pac J Cancer Prev 16:1139-1143.

Zhu XJ, Liu J, Xu XY, Zhang CD, and Dai DQ. 2014. Novel tumor-suppressor gene epidermal growth factorcontaining fibulin-like extracellular matrix protein 1 is epigenetically silenced and associated with invasion

Peer] reviewing PDF | (2019:04:36425:1:1:NEW 31 May 2019) 


\section{Table $\mathbf{1}$ (on next page)}

The detail information of four GEO datasets 
Table 1 The detail information of four GEO datasets

\begin{tabular}{ccccc}
\hline ID & Tissue & Platform & Normal & Tumor \\
\hline GSE118370 & LUAD & GPL570 & 6 & 6 \\
GSE85841 & LUAD & GPL20115 & 8 & 8 \\
GSE43458 & LUAD & GPL6244 & 30 (never-smoker) & 40 (never-smoker) \\
GSE32863 & LUAD & GPL6884 & 58 & 58 \\
\hline
\end{tabular}

GEO: Gene Expression Omnibus; LUAD: Lung Adenocarcinoma 
Table 2 (on next page)

Top ten up- and down-regulated DEGs 
Table 2 Top ten up- and down-regulated DEGs

\begin{tabular}{|c|c|c|c|c|c|}
\hline \multicolumn{3}{|c|}{ Up-regulated DEGs } & \multicolumn{3}{|c|}{ Down-regulated DEGs } \\
\hline Gene symbol & $\log 2 \mathrm{FC}$ & FDR & Gene symbol & $\log 2 \mathrm{FC}$ & FDR \\
\hline SPP1 & 3.08474 & $1.74 \mathrm{E}-33$ & FABP4 & -3.37555 & $1.25 \mathrm{E}-47$ \\
\hline OCIAD2 & 1.477746 & $1.13 \mathrm{E}-29$ & STX11 & -2.01766 & $1.51 \mathrm{E}-44$ \\
\hline ETV4 & 1.366623 & $5.38 \mathrm{E}-27$ & CAV1 & -2.70539 & $6.04 \mathrm{E}-43$ \\
\hline TOP2A & 1.777989 & $3.24 \mathrm{E}-26$ & FHL1 & -2.3099 & $5.77 \mathrm{E}-42$ \\
\hline COL10A1 & 1.364488 & $3.74 \mathrm{E}-26$ & TEK & -2.30698 & $1.09 \mathrm{E}-40$ \\
\hline PROM2 & 1.570558 & $7.77 \mathrm{E}-26$ & AGER & -2.69928 & $6.05 \mathrm{E}-40$ \\
\hline MMP11 & 1.929847 & $1.03 \mathrm{E}-23$ & FMO2 & -2.44791 & $1.42 \mathrm{E}-39$ \\
\hline UBE2T & 1.154061 & $5.78 \mathrm{E}-23$ & CRYAB & -1.98382 & $1.77 \mathrm{E}-39$ \\
\hline $\mathrm{ABCC} 3$ & 1.444218 & $3.71 \mathrm{E}-22$ & GRK5 & -1.4854 & $1.53 \mathrm{E}-38$ \\
\hline BAIAP2L1 & 1.067336 & $4.15 \mathrm{E}-22$ & TMEM100 & -2.928 & 4.79E-38 \\
\hline
\end{tabular}

1 DEGs: Differentially Expressed Genes; FDR: False Discovery Rate; Log2FC: Log2(Fold Change) 
Table 3 (on next page)

KEGG enriched pathways of DEGs 
Table 3 KEGG enriched pathways of DEGs

\begin{tabular}{clcc}
\hline \multicolumn{1}{c}{ ID } & \multicolumn{1}{c}{ Description } & Count & p.adjust(FDR) \\
\hline hsa04610 & Complement and coagulation cascades & 13 & $1.12 \mathrm{E}-05$ \\
hsa05144 & Malaria & 9 & 0.000271 \\
hsa05020 & Prion diseases & 7 & 0.001406 \\
hsa05418 & Fluid shear stress and atherosclerosis & 13 & 0.001921 \\
hsa04933 & AGE-RAGE signaling pathway in diabetic & 10 & 0.007362 \\
hsa04270 & Complications & 11 & 0.014433 \\
hsa04657 & Vascular smooth muscle contraction & 9 & 0.014433 \\
hsa04974 & Protein digestion and absorption & 8 & 0.039299 \\
hsa04670 & Leukocyte transendothelial migration & 9 & 0.039299 \\
hsa00982 & Drug metabolism - cytochrome P450 & 7 & 0.039299 \\
\hline
\end{tabular}

1 KEGG: Kyoto Encyclopedia of Genes and Genomes; DEGs: Differentially Expressed Genes; FDR: False 2 Discovery Rate 
Figure 1 (on next page)

Volcano plot of the DEGs.

The vermilion and blue dots represent DEGs filtered based on the cut-off values of $\mid \log 2$ FoldChange $>1.0$ and adjusted $P$ value $(F D R)<0.05$, while the black dots represent genes that are not satisfied the cut-off values of differential expression. The horizon dotted line indicates the position of $-\log 10(F D R)=0.05$, and the vertical dotted lines indicate the positions of $\mid \log 2$ FoldChange $=1.0$. Vermilion : upregulation; Blue: downregulation. DEGs: differentially expressed genes. FDR:False Discovery Rate. 
PeerJ

Volcano Plot of DEGS $\mathcal{S}^{\text {cript to be reviewed }}$

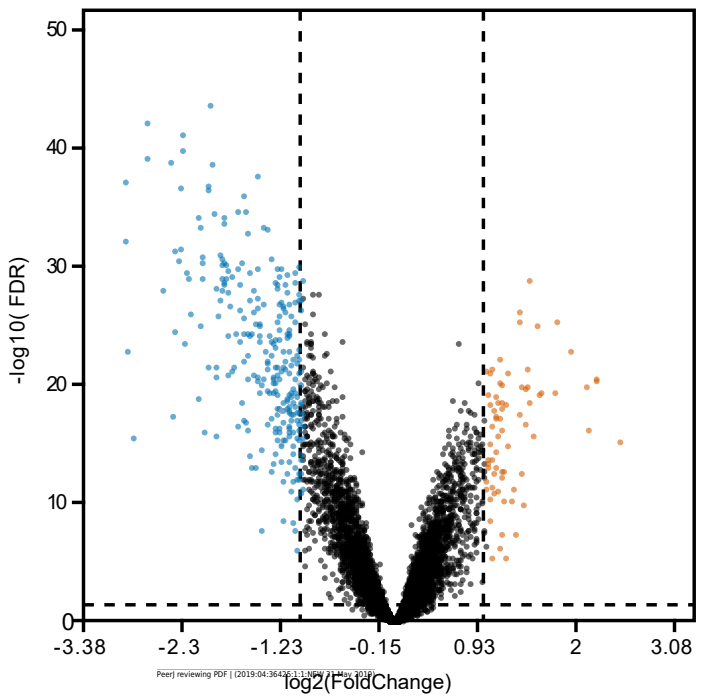


Figure 2 (on next page)

GO and KEGG analysis of DEGs.

(A-C) The top 15 terms of $\mathrm{GO}$ categories of biological process (BP), cellular component (CC) and molecular function MF, respectively. (D) KEGG pathway analysis of DEGs, p.adjust(FDR) $<0.05$ was considered significantly. GO: Gene Ontology; KEGG: Kyoto Encyclopedia of Genes and Genomes;FDR:False Discovery Rate. 


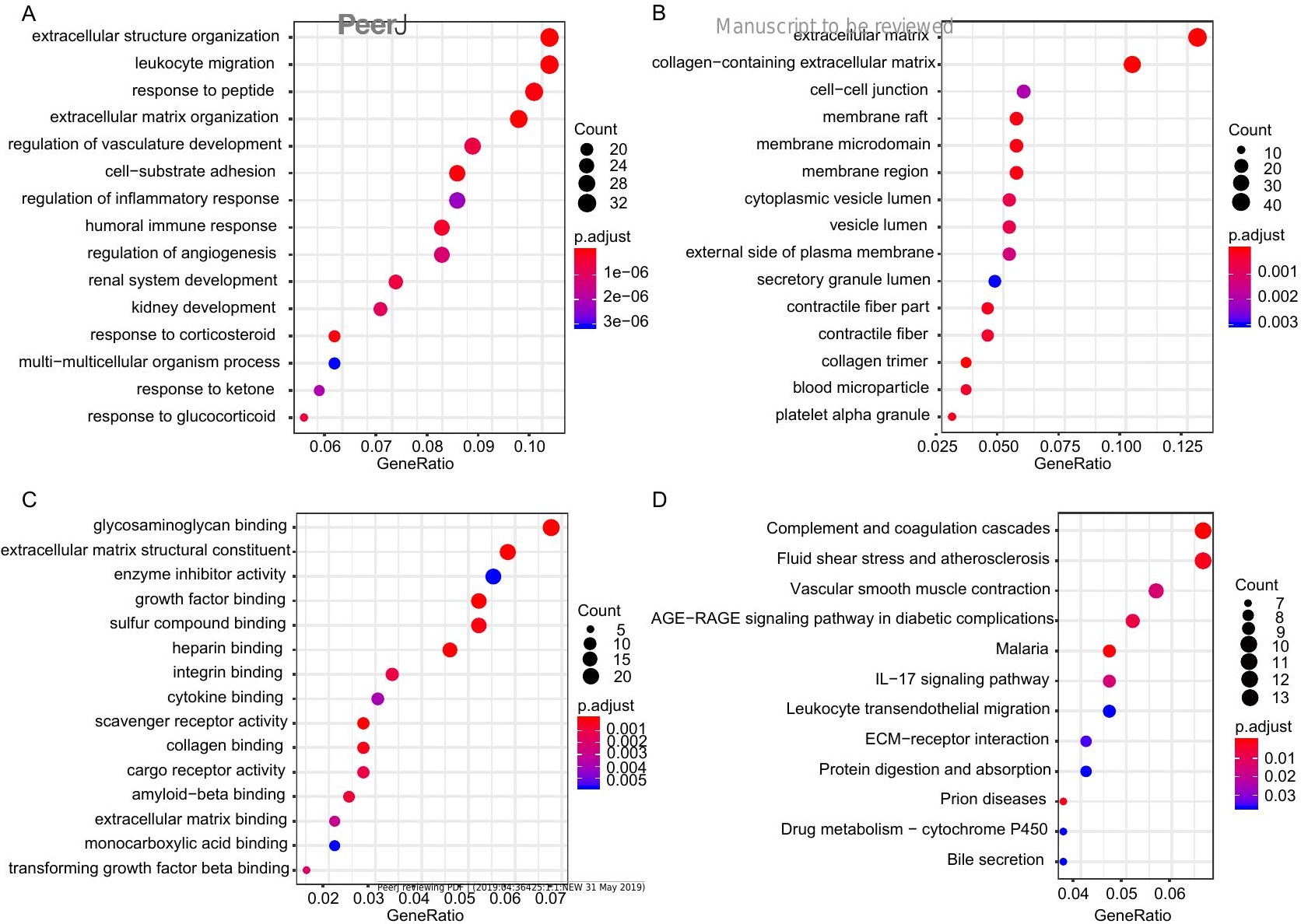


Figure 3 (on next page)

Protein-protein interaction network of DEGs and modules analysis.

(A) PPI network of DEGs; (B) The KEGG enrichment analysis of the genes in the top 3

modules. (C-E) the top 3 modules of PPI network. The red nodes represent the upregulated DEGs. The green nodes represent the downregulated DEGs. PPI: protein-protein interaction; DEG: differentially expressed gene; KEGG: Kyoto Encyclopedia of Genes and Genomes. 

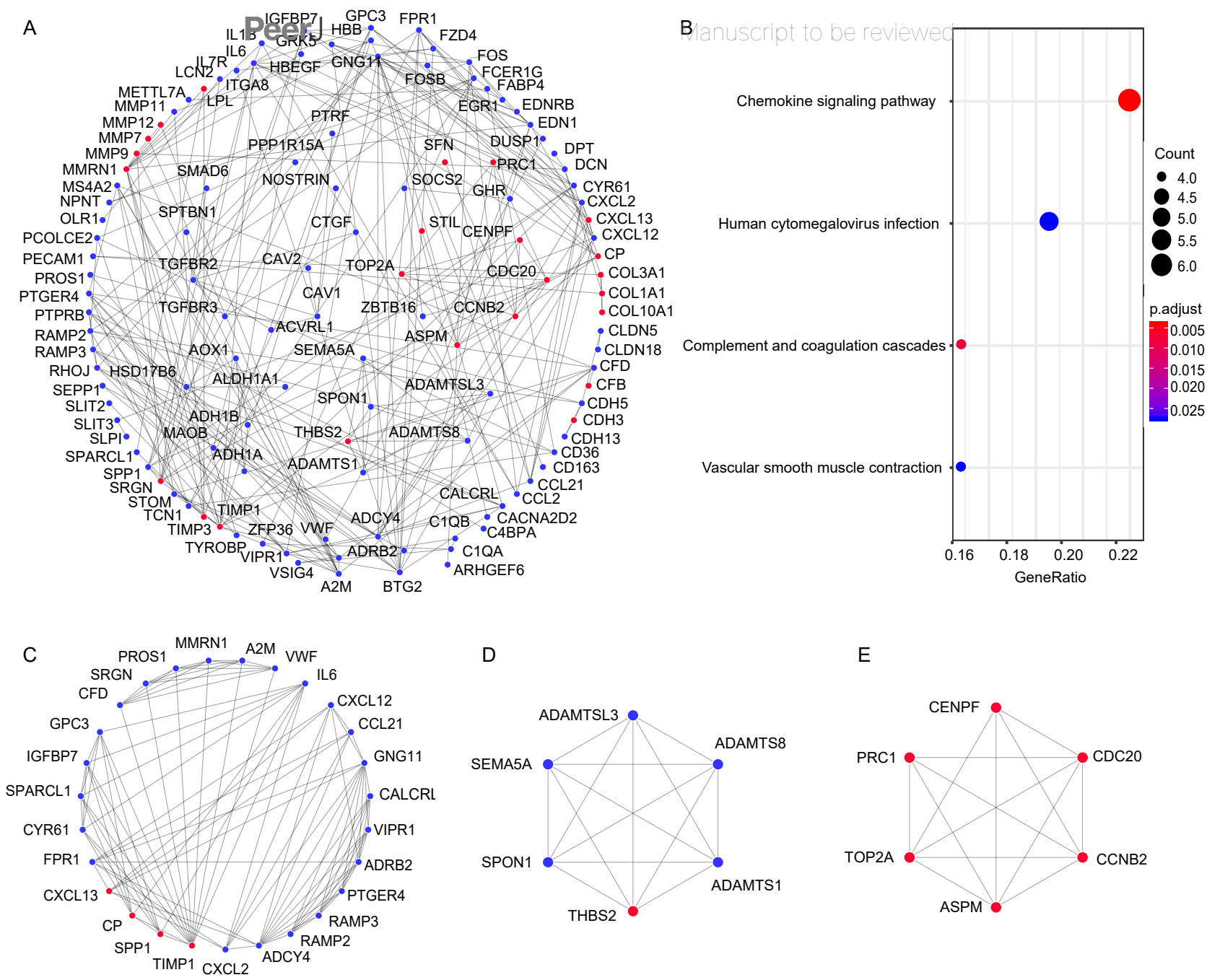

E

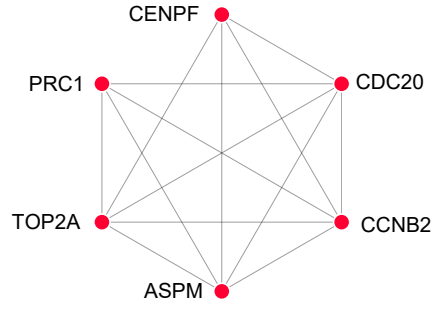


Figure 4 (on next page)

Overall survival analyses of hub genes.

(A-J) The overall survival analyses of hub genes were performed using Kaplan- Meier Plotter online platform. Logrank $\mathrm{P}<0.05$ was considered statistically significant. 
Figure $\mathbf{5}$ (on next page)

Oncomine analysis of cancer vs. normal tissue of ADAMTS8, METTL7A , MYH10 and PRC1.

Heat maps of ADAMTS8(A), METTL7A(B), MYH10(C) and PRC1(D) gene expression in lung adenocarcinoma samples vs. normal tissues. 1. Lung Adenocarcinoma vs. Normal, Hou Lung (Hou et al. 2010) ; 2. Lung Adenocarcinoma vs. Normal, Landi Lung (Landi et al. 2008) ; 3. Lung Adenocarcinoma vs. Normal, Selamat Lung (Selamat et al. 2012) ; 4. Lung Adenocarcinoma vs. Normal, Su Lung (Su et al. 2007) . 
A

\begin{tabular}{|rrr|r|r|r|r|}
\hline Median Rank & P-Value & \multicolumn{2}{l|}{ Gene } \\
\hline 79.5 & $7.34 \mathrm{E}-28$ & ADAMTS8 & & & & \\
& & & 1 & 2 & 3 & 4 \\
\cline { 3 - 8 } & & & &
\end{tabular}

1. $P$-value $=5.54 E-27$ FoldChange $=-6.612$

2. $P$-value $=1.47 \mathrm{E}-27$ FoldChange $=-2.990$

3. $P$-value $=9.12 E-38$ FoldChange $=-2.848$

4. $P$-value $=1.81 \mathrm{E}-11$ FoldChange $=-8.009$
B

\begin{tabular}{|c|c|c|c|c|c|c|}
\hline Median Rank & P-Value & Gene & & & & \\
\hline 547.5 & 1.13E-9 & METTL7A & & & & \\
\hline & & & 1 & 2 & 3 & 4 \\
\hline
\end{tabular}

1. $P$-value $=8.55 E-13$ Fold Change $=-2.482$

2. $P$-value $=2.26 \mathrm{E}-28$ FoldChange $=-3.213$

3. $P$-value $=8.11 \mathrm{E}-17$ FoldChange $=-2.522$

4. $P$-value $=2.25 E-9$ FoldChange $=-3.382$
C

\begin{tabular}{|c|c|c|c|c|c|c|}
\hline Median Rank & P-Value & Gene & & & & \\
\hline \multirow[t]{2}{*}{235.5} & 1.07E-23 & MYH10 & & & & \\
\hline & & & 1 & 2 & 3 & 4 \\
\hline
\end{tabular}

1. $P$-value $=4.97 E-17$ FoldChange $=-2.673$

2. $P$-value $=2.15 E-23$ FoldChange $=-2.649$

3. $P$-value $=1.79 E-26$ FoldChange $=-2.797$

4. $P$-value $=1.72 E-12$ FoldChange $=-3.491$

D

$\begin{array}{rrr}\text { Median Rank } & \text { P-Value } & \text { Gene } \\ 220.5 & 5.56 \mathrm{E}-7 & \text { PRC1 }\end{array}$

PRC1

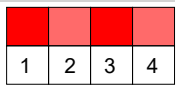

1. P-value $=2.41 \mathrm{E}-17$ FoldChange $=4.260$

2. $P$-value $=1.88 \mathrm{E}-14$ FoldChange $=2.661$

3. P-value $=1.16 \mathrm{E}-19$ FoldChange $=2.677$

4. P-value $=1.11 \mathrm{E}-6$ FoldChange $=2.610$ 
Figure 6 (on next page)

The association between the expression of selected hub genes and tumor stage.

(A-D) The expressions of ADAMTS8, METTL7A, MYH10, PRC1 were correlated with tumor stage in the Okayama Lung dataset. 0: No value; 1 : Stage I; 2: Stage II. 


\section{$\mathrm{A}$}

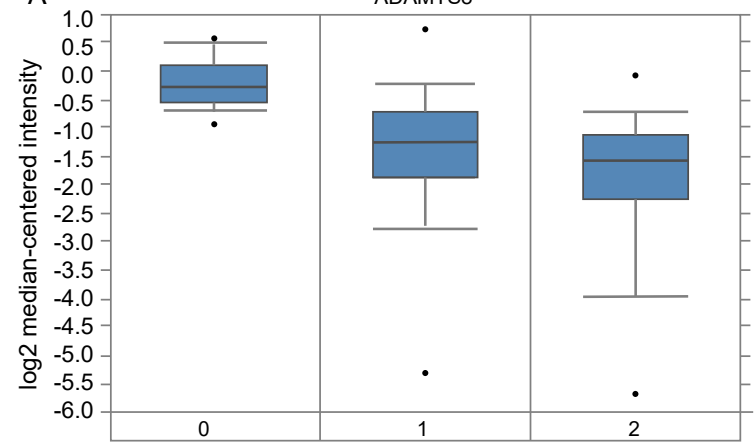

C

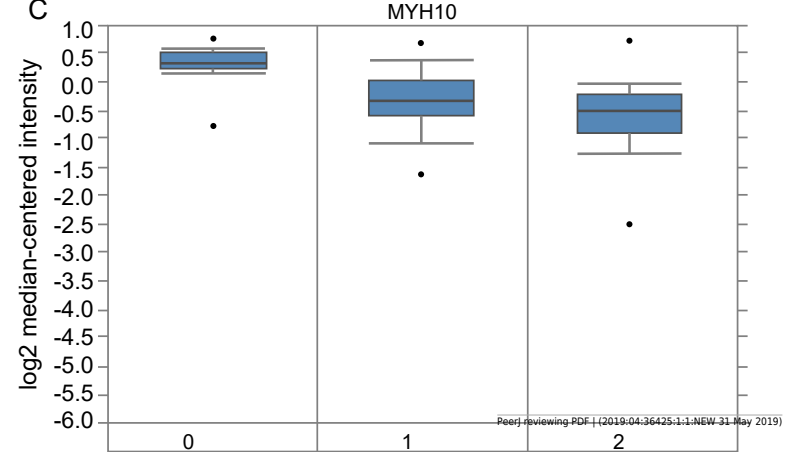

B Manuscript to be reviewed

METTL7A

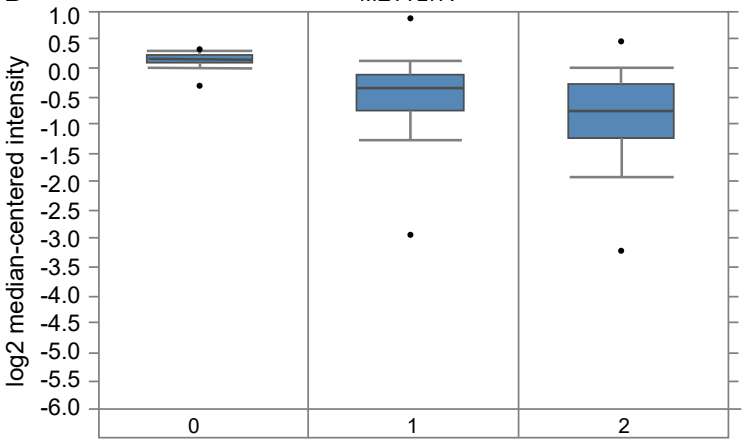

D

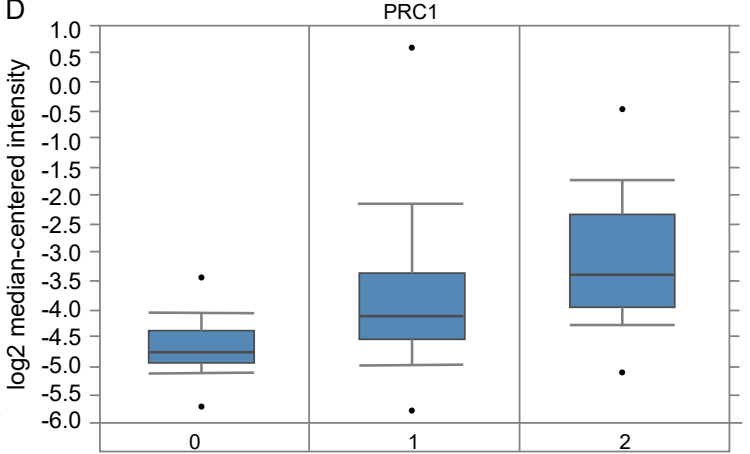

